for dying patients in need of care which was inaccessible in the community.

\section{RENAL REPLACEMENT THERAPY IN PATIENTS APPROACHING THE END OF LIFE: A CASE SERIES OF 3 PATIENTS WHO MADE DIFFERENT CHOICES}

Zoe Kantor, Aaron Braddy, Andrew Findlay. Department of Renal Medicine, East and North Herts NHS Trust, The Lister Hospital, Stevenage, UK

\subsection{6/bmjspcare-2018-ASPabstracts.97}

Objective To explore decisions regarding the use renal replacement therapy (RRT) as patients approach end of life.

Background End stage renal failure (ESRF) has many potential treatment options, including RRT, renal transplantation and conservative management. Complexities exist (including the impact on quality of life and length of life) regarding the instigation or withdrawal of RRT in patients who are nearing end of life. RRT is unique as no other organ function can be as readily and effectively replaced; this alone can make it difficult for people to choose not to have treatment.

Method We reviewed three cases of patients with ESRF approaching the end of life:

Patient 1 - On renal replacement therapy, wishing to withdraw from RRT

Patient 2 - A patient with a short life expectancy due to comorbidities but who wanted to commence RRT for acuteon-chronic renal failure who developed immediate cardiac complications requiring end of life care

Patient 3 - A patient who was being conservatively managed who then developed acute-on-chronic renal failure due to an acute illness who declined RRT

The role of the multi-disciplinary team in the decision-making process, in conjunction with discussions with patients and their relatives, was also considered.

Results The three patients made different choices for the management of their renal failure as they approached end of life. They made informed decisions about their care with the support their family and the renal multi-disciplinary team.

Lessons learnt The impact of RRT on quality and length of life should be considered in patients nearing the end of their lives. Renal MDT involvement in the decision-making process is imperative to ensure the patient makes an informed decision. The three patients considered made different choices as they approached end of life reminding us of the importance of patient's wishes in their end of life planning.

\section{THE ROLE OF NURSES IN CARE OF THE DEAD PERSON}

Christina Faull, Lindsey Buster, Laura Green, Karina Croucher. LOROS Hospice, Leicester, University of Bradford

\subsection{6/bmjspcare-2018-ASPabstracts.98}

Background The care of a person who has died is of importance to the person themselves and to their loved ones. Nurses have a unique role in providing this care. This work explored the literature concerning the formal practices of the care of a dead person, the teaching of such practices and the cultural and informal curriculum by which such practices are passed on in nursing.
Methods A pragmatic review of UK and US published literature since the establishment of nursing as a trained profession in 1860. Journal databases, textbooks of nursing as well as historical archives were searched for materials.

Results The care of the dead person is ritualistic and prescribed. There are strong cultural influences on practices and little evidence base upon which these practices are based. Geo-political, religious and local cultures all have strong influences in both the practices and the tone of the care. The informal or tacit curriculum, such as the placement of tokens of respect such as flowers or the opening of windows to 'let the spirit out' is absent from teaching materials but alluded to in memoires and personal accounts.

The care of the dead person is secretive and professionally guarded. By some it is seen as a privileged activity and by others as an activity akin to punishment or of low value in the work of that shift, even one to avoid.

Conclusions The care of the dead person is an area of secrets and diversity of cultural values and approaches. There is a paucity of literature and evidence base.

\section{TALKING RESUSCITATION - CAN WE GET IT RIGHT AT THE HOSPICE?}

Alexandra Rusby, Aruna Hodgson. Wigan and Leigh Hospice

\subsection{6/bmjspcare-2018-ASPabstracts.99}

Background There have been a number of high profile cases about resuscitation decisions in recent years. ${ }^{1,2}$ This has prompted regular updates in the national guidance on decisions relating to cardiopulmonary resuscitation (CPR). ${ }^{3-5}$ At the hospice a specific template is used to document CPR decisions on SystmOne, electronic patient record system. Current practice on the inpatient unit was audited against the hospice resuscitation policy following local and national guidance. ${ }^{3,6}$ Fourteen standards were set specifically reviewing documentation on admission, at multidisciplinary team meetings and discharge. A $100 \%$ target was set for each standard due to the important implications of the do not attempt cardiopulmonary resuscitation (DNACPR) decision. The aim was to establish whether CPR decisions were documented and communicated appropriately to highlight any areas for improvement.

Method Retrospective review of 39 patient notes on SystmOne for patients admitted to the hospice from October to November 2016.

Results Of the fourteen standards set, only one standard met the $100 \%$ target. This was informing the GP of the unified DNACPR (uDNACPR) status at discharge.

Standards that achieved over $90 \%$ included: making a resuscitation decision on admission and discussing this with the patient, as well as reviewing resuscitation status at multidisciplinary team meetings.

Standards that achieved less than 80\% included: discussing resuscitation decision with relatives when the patient lacks capacity and documenting decisions for uDNACPR at discharge.

Conclusion Using SystmOne templates has been helpful for documenting CPR status on admission, at multidisciplinary meeting review and on discharge letters to the GP. Recommended amendments to the CPR template include a prompt for a uDNACPR decision at discharge and an updated discharge checklist. 
It is recommended that doctors fully document the CPR discussions when completing these templates even if the uDNACPR is in place, especially with relatives of patients without capacity.

\section{\begin{tabular}{l|l}
73 & INTRODUCTION OF A COMMUNITY PALLIATIVE CARE
\end{tabular} DRUG CHART TO FACILITATE INDIVIDUALISED AND APPROPRIATE ANTICIPATORY PRESCRIBING}

Candida Cornish, Sarah French. St. Peter's Hospice, Bristol Clinical Commissioning Group, Bristol North Somerset South Gloucestershire Anticipatory Prescribing Working Party

\subsection{6/bmjspcare-2018-ASPabstracts. 100}

Background NICE guidelines stress the importance of individualised anticipatory prescribing for people who are likely to need symptom control in the last days of life. A Community Palliative Care Drug Chart for the authorisation of individualised subcutaneous palliative care medication via injection or syringe pump was developed and introduced by a regional anticipatory prescribing working party. The chart does not use a proforma approach, which has been linked to over medication, but does incorporate guidance and is designed to cross settings.

Aim

- To evaluate whether the chart and guidance facilitates safe, appropriate and consistent anticipatory prescribing for patients 1 year after introduction.

- To identify any required chart adaptations and areas for education.

Methods The notes of 49 expected deaths from District Nurse caseloads were examined for the presence of a chart. 32 charts were reviewed to establish whether anticipatory prescribing complied with local guidelines and to record any themes from non-compliance. A survey of 20 local GPs was conducted.

Results 47 of the 49 expected deaths had a chart in place. For as required medication the percentage meeting the standards for choice of drug, dose and route were as follows: opioid (84\%), antiemetic (97\%), antisecretory (94\%) and anxiolytic (94\%). In 7 cases the range for opioid or midazolam in a syringe pump was greater than advised in guidelines. All GPs surveyed agreed that the chart facilitates safe and appropriate anticipatory prescribing.

Conclusion The chart and guidance facilitate safe, appropriate and consistent anticipatory prescribing for patients, resulting in improved compliance with standards when compared to a local audit from 2010. Changes to the chart and further education is needed in relation to syringe pump ranges and timing of authorisation. We have subsequently developed a new 'intelligent' protocol within the EMIS GP record to further enhance individualised anticipatory prescribing.

\section{ANTICIPATORY PRESCRIBING IN PALLIATIVE CARE: EVALUATION OF CURRENT PRACTICE}

Abiramithevi Ponnampalampillai, Ros Marvin, Sarah Grove, Stephen Barclay, Anna Spathis. Cambridge University Hospitals NHS Trust, University of Cambridge

\subsection{6/bmjspcare-2018-ASPabstracts.101}

Background National guidelines endorse prescription of medications in anticipation of symptoms in the last days of life.
Little is known about current practice in anticipatory prescribing (AP). A service evaluation was undertaken to evaluate $\mathrm{AP}$ across one county in the UK.

Method Electronic records were searched of patients known to the Bedfordshire PEPS (Partnership for Excellence in Palliative Support) Co-ordination Centre, who had died during a one-year period and had been prescribed injectable medications for symptom control.

Results Out of 392 eligible patients, the records of 132 were selected at random. 486 injectable medications were prescribed: $77 \%$ were in anticipation of symptoms and $23 \%$ in response to existing symptoms. Diamorphine and midazolam were most commonly prescribed in anticipation, with 43/88 (49\%) and 41/93 (44\%) prescriptions leading to drug administration respectively. The corresponding data for glycopyrronium, cyclizine, haloperidol and metoclopramide were 25/78 (32\%), 15/67 (22\%), 3/11 (27\%) and 3/14 (21\%). Overall, only $37 \%$ of all medications prescribed in anticipation were administered. Recognition of the need for AP came from palliative care nurses (50\%), GPs (32\%) and District Nurses (14\%).

Most patients had malignant disease (87\%). The median time between prescription and first drug administration was 9 days in patients with cancer (range 0-368), and 61 days in those with non-malignant disease (range 3-298).

Conclusion Most prescribing of injectable medications was in anticipation, rather than in response to, symptoms. About a third of anticipatory prescribing led to drug administration. Although diamorphine and midazolam were both commonly prescribed and administered, antiemetics were less so. Further work is needed to evaluate the cost-effectiveness of AP, particularly in relation to antiemetics, and to investigate the unexpectedly long interval between drug prescription and administration in patients with non-malignant disease.

\section{SYMPTOM MANAGEMENT AT THE END OF LIFE: AN EVALUATION OF DOCUMENTED RESPONSE POST- ADMINISTRATION OF ANTICIPATORY MEDICATIONS IN A DISTRICT GENERAL HOSPITAL}

Joanne Hayes, Natalie Wright, Susan Dargan, Clare Smith. Ashford and St. Peter's NHS Foundation Trust

\subsection{6/bmjspcare-2018-ASPabstracts. 102}

Background The standard of practice for end of life care in the UK is to use anticipatory medications for symptom control. Given this it is important for the benefits or side effects of the medication to be identified so they can be adjusted to meet the patient's needs.

Aim To assess how frequently the response to anticipatory medications was documented by nursing staff, in both general wards and specialist palliative care beds at a district general hospital.

Methods A retrospective audit was conducted at a district general hospital in the UK. All palliative care referrals during October 2016 were audited for evidence of anticipatory medication use and whether there was any nursing documentation of its effectiveness. This was also done for all patients admitted to the newly-established specialist palliative care beds at the same hospital over a three month period in 2017.

Results 102 patients were referred to palliative care in October 2016, of which 50 had anticipatory medications given. Of 